\title{
INTERACCIONES CONTEMPORÁNEAS: EL PATRIMONIO EN LOS MEDIOS DE COMUNICACIÓN
}

\author{
Modesto García-Jiménez1: Universidad Católica San Antonio. España \\ mgarcia@pdi.ucam.edu
}

\section{RESUMEN}

No cabe duda de que todo el enorme fenómeno social, cultural, político económico y mediático al que solemos referirnos bajo la denominación genérica de Patrimonio, es uno de los más importantes de la actualidad en todo el mundo. Las pruebas de ello son contundentes: basta con echar un vistazo a los grandes proyectos nacionales, que casi siempre tienen como referente principal el Patrimonio y su relación con las estrategias de crecimiento económico y de desarrollo. Históricamente, el Patrimonio se ha constituido como uno de los pilares básicos de la construcción del Estado y es indudablemente un referente de primer orden en lo que respecta a la identidad y a las aspiraciones de simbolismo colectivo de todos los pueblos y culturas. La gigantesca importancia de la dimensión procedimental del Patrimonio, oculta a su vez la necesaria generación teórica y su ubicación y justificación en los modos de comportamiento social. Es en la proyección que de estos asuntos hacen los medios de comunicación de masas donde realmente se construye la enorme envergadura del fenómeno y sus sólidos anclajes en el Estado, la identidad y el desarrollo. Esta comunicación quiere arrojar luz sobre la importancia, en este contexto, de la labor de los medios de comunicación.

PALABRAS CLAVE: Patrimonio - Estado - ciencia - medios de comunicación social crítica

\footnotetext{
${ }^{1}$ Autor correspondiente

Modesto García-Jiménez: Universidad Católica San Antonio. España

Correo: mgarcia@pdi.ucam.edu
} 


\title{
CONTEMPORARY INTERACTIONS: HERITAGE IN THE MASS MEDIA
}

\begin{abstract}
There is no doubt that the whole social, cultural, political and economic and media phenomenon, which we usually refer to under the term heritage, is currently one of the most relevant over the world. Evidences of this fact are plausible: it is enough to have a look at the great national projects, which mainly relate almost always to heritage and its relation to the strategies of economic and development growth. Historically, heritage has been established as one of the basic milestones of national construction and is undoubtedly a chiefly reference regarding identity and the ambitions of the collective symbolism both of peoples and cultures. The giant importance of the procedure dimension of heritage hides in turn the necessary generation of theories and its location and justification through the ways of social behaviour. It is in the projection of these matters by mass media when the enormous span of this phenomenon is really built up, as well as its solid hooks in nations, identity and development. This paper aims to shed light on the importance, in this context, of the task of mass media.
\end{abstract}

KEY WORDS: Heritage - State - Science - Mass Media - Critical Perspective

\section{INTRODUCCIÓN}

No cabe duda de que todo el enorme fenómeno social, cultual, político económico y mediático al que solemos referirnos bajo la denominación genérica de Patrimonio, es uno de los más importantes de la actualidad en todo el mundo. Las pruebas de ello son contundentes: basta con echar un vistazo a los grandes proyectos nacionales -y por ende, regionales y locales-, que casi siempre tienen como referente principal el Patrimonio y su relación con las estrategias de crecimiento económico y desarrollo.

Históricamente, el Patrimonio se ha constituido como uno de los pilares básicos de la construcción del Estado (desde los escritos de A.-Ch. Quatremère de Quincy [1796]), aunque en la producción crítica actual sobre la temática se apunte una importante e interesante derivación hacia la construcción del Estado Cultural (tal y como lo expone M. Fumaroli [1992]); es indudablemente un referente de primer orden en lo que respecta a la identidad y a las aspiraciones de simbolismo colectivo de todos los pueblos y culturas; y, en estos mismos sentidos, se erige en la sociedad contemporánea como uno de los más claros contextos de lo que entendemos por globalización, si se tiene en cuenta la existencia de instituciones supranacionales de tratamiento específico del Patrimonio (UNESCO). 
La gigantesca importancia de la dimensión procedimental del Patrimonio, oculta a su vez la necesaria generación teórica y su ubicación y justificación en los modos de comportamiento social, y elimina casi por completo las voces críticas que pudieran existir, a pesar de que éstas son, de alguna manera, imprescindibles, cuando se trata de fenómenos sociales de tan aparentes bondades y contra los que nadie osa posicionarse (J. Clifford, 2003, p. 17); (V. Padiglione, 1999, p. 22). Evidentemente, el Patrimonio, su gestión, sus procedimientos, la distribución de sus fondos, la justificación de sus criterios de catalogación, la arbitrariedad de sus elecciones, etc., crean márgenes sociales, o en cualquier caso producen un tipo de segregación social.

La producción científica en torno al Patrimonio está más inclinada al tratamiento de sus procedimientos y realizaciones que a la producción teórico-crítica sobre su efecto social y cultural. Pero es en la proyección que de estos asuntos hacen los medios de comunicación de masas donde realmente se construye la enorme envergadura del fenómeno y sus sólidos anclajes en el Estado, la identidad y el desarrollo.

Esta comunicación quiere arrojar luz sobre cómo es que la labor difusora de los medios de comunicación se convierte, en esta tesitura, en elemento fundamental, que tendrá en algunos casos que renunciar a la 'vistosidad' y espectacularidad de las grandes realizaciones en el campo patrimonial, en beneficio de su cabal entendimiento social, de sus débitos ideológicos, de clientelismo político, enfrentamientos o exclusiones identitarios, etc.

\section{DESARROLLO}

\subsection{Del Patrimonio, otra vuelta de tuerca}

Una de las estrategias teóricas de mayor repercusión en el contexto de estudios sobre el Patrimonio ha sido, junto con la de competencia, la de mediación. Cierto es que éstas se insertan en una coyuntura en la que las lecturas más críticas sobre el gran fenómeno cultural de nuestros días -me refiero a este del Patrimonio- todavía no habían conseguido lograr un gran eco en el conjunto teórico. A decir verdad, todavía no lo han conseguido.

Las nociones de competencia y mediación aplicadas a las perspectivas de Patrimonio hacían gala de cierta bondad en la propuesta. En absoluto suponían ningún tipo de planteamiento crítico más allá de los aspectos más ásperos que encerraban en el desglose de sus propios postulados. 
Puede decirse que, de principio, no suponían un posicionamiento crítico, como lo es por ejemplo la teoría del Estado Cultural (Fumaroli, 1992) u otras que parten del hecho de tener en cuenta la arbitrariedad de los procedimientos patrimoniales -sobre todo en lo que se refiere a las mecánicas de catalogación por parte de instituciones supranacionales- u otras cuestiones más sangrantes como la exclusión social que como referente identitario para unos grupos necesariamente provocan con respecto a otros, o el en cierta manera escarnio que puede suponer considerar 'propietarios' de cierto patrimonio -evidentemente en su versión más metafórica- a grupos humanos que literalmente mueren de hambre o de otras calamidades.

El término operativo de competencia nace en una tradición en la que todavía los aspectos procedimentales no se habían alejado en demasía del interés común; es decir, se trataba todavía de un asunto en ciernes aunque ya se vislumbraba la enorme envergadura del fenómeno en el que de inmediato se iba a convertir. La competencia, entonces, nadaba entre las aguas de la necesidad de desvelar y transmitir las presuntas raíces de esencia social del Patrimonio, en lo que a constituirse como un verdadero referente identitario y de progreso se refiere, y por otro lado la necesidad también de que el Estado se erija como responsable de la catalogación, tratamiento y gestión de los elementos patrimoniales y de los recursos que de su puesta en valor se derivan. Se trata de un doble sentido de competencia. Uno es científico y socioantropológico; el otro, civil, institucional y estatutario.

El de mediación es igualmente complejo, si no más. En un primer supuesto, los agentes competentes en la gestión y tratamiento del Patrimonio establecen una relación que se pretende idónea entre éste y sus depositarios, propietarios, protagonistas... o cualquier otra de las numerosas apelaciones más o menos eufemísticas que a los referenciados en el Patrimonio se les da en la ya numerosa producción científico intelectual que sobre el caso existe.

Es evidente que como fenómeno privilegiado de la Modernidad (como ya se ha indicado, desde la consolidación del Estado, cuando no de su propia creación, hasta la conformación de un imaginario social, la construcción muchas veces de la Historia o la constitución como referente identitario de los grupos humanos, por no hablar de su dimensión económica que es en cierto modo incuestionable) el Patrimonio en sus campos teórico y procedimental está expandiendo sus propios contornos hasta posicionarse, impregnar, interesar todos los aspectos, ya de política cultural como de política económica.

La tendencia según algunos tratadistas de la cuestión es un avance hacia establecer su presencia en todos los campos sociales, utilizando para ello la versatilidad que le otorga el hecho de que en realidad todo es herencia y por ello todo es patrimonio (Neyret, 2004, p. 5), y mucho más la que conlleva las enormes posibilidades de expansión abiertas con las nuevas consideraciones en torno al 
patrimonio intangible o inmaterial.

En efecto, las políticas patrimoniales parecen dirigirse hacia un 'todo patrimonio', una especie de metástasis de 'bien cultural' que única y necesariamente se va a ordenar según el 'turno' de catalogación que cada uno de los elementos candidatos obtenga de las instituciones supranacionales o, en su caso, nacionales, regionales, etc. Lógicamente, este desbordamiento provoca reacciones en cierta manera enfrentadas. Por un lado toda pequeña o gran comunidad reivindica su derecho a que 'sus patrimonios' sean reconocidos y sancionados -corroborados- según las exigencias de la política competente.

Ello puede arrastrar una reinvención continua de patrimonios y herencias culturales. En el lado contrario, la necesidad de poner en marcha procesos de consenso automáticos, que en realidad van a responder a intereses muy diversos y muy arbitrarios, va a provocar a su vez rupturas constantes de ese consenso. Lo que para unos es incuestionablemente patrimonio para otros no lo es con tanta claridad o incluso es deleznable como herencia y por ello es deseable su exclusión de las glorias patrimoniales (Cobo de Guzmán, 2010, p. 447).

Esta situación, que se extiende por todos los niveles administrativos e institucionalesdesde el internacional hasta el local-, requiere de la todavía no acabada noción de 'mediación' una reactivación y una readecuación de sus herramientas conceptuales y de procedimiento. O podríamos decir que la estrategia de la mediación sufre una transmutación de su sentido al enfrentarse a esta expansión desbordante de 'los patrimonios'; lo que era prácticamente una labor casi exclusivamente de información se va a convertir en una compleja trama de 'tratos' y de captación de voluntades, cuando no en una especialidad de reparación de agravios.

En algunas tradiciones teóricas de tratamiento del Patrimonio -fundamentalmente en la italiana (Padiglione, Clemente, Lattanzi, Portelli)- esta transmutación se va a convertir pronto en un significativo reto sociantropológico, el que paulatinamente va de las formas políticas de mediación a las de cualidad estratégica de 'negociación'. Efectivamente, el cambio es altamente significativo en el seno de la filosofía de las herencias culturales e históricas, pues se trata, como no es difícil apreciar, de una derivación cualitativa encaminada a considerar a los protagonistas del Patrimonio como sujetos portadores de conocimiento y criterio, que desde sus posiciones ideológicas respectivas van a revolucionar el orden establecido en la catalogación patrimonial, al invertir el sentido de la propuesta que en adelante va a seguir un orden más acorde con lo que pudiéramos llamar la razón patrimonial: de abajo a arriba, y no, como siempre sucede, al contrario.

Conviene matizar que frente, o paralelamente, a la propuesta italiana, en cierta manera impregnada de las últimas teorías de corte dialéctico histórico, pueden situarse otras de parecido fondo teórico pero quizá con menor énfasis en sus terminologías. 
Éstas, puede que abanderadas por J. Clifford, proponen una 'antropología compartida', es decir, la creación de un contexto perceptivo del patrimonio que se elabora desde abajo -desde las bases diría la tradición italiana- para lograr un consenso en torno a qué es Patrimonio y a quiénes representa simbólicamente, y como es lógico también en qué términos se articula su dimensión de recurso económico y de desarrollo, sobre todo para evitar contradicciones y situaciones paradójicas insostenibles como puede ser la recuperación del patrimonio indígena como nuevo valor sentimental para los estadounidenses pero sin enmendar y a veces sin ni siquiera retomar el debate de las reservas indias y su situación deleznable (Clifford, 2010, p. 264). O en un entorno más cercano la patrimonialización de los marcos geográficos, laborales y sociales de la minería giennense sin haber solucionado satisfactoriamente sus graves secuelas socioprofesionales (Cobo de Guzmán 2010, p. 454).

Parece estar claro que en su sentido moderno el Patrimonio pasa de ser una predeterminada herencia -en cierta forma fatídica- a convertirse en un recurso social, que en el más optimista de sus enfoques construimos y reconstruimos constantemente, haciendo de él un referente de expectativas de progreso. Sin embargo a veces la realidad es otra bien distinta: es históricamente una herramienta fundamental para la consolidación, incluso para la creación del Estado (Højrup 2010, p. 303); y en el tiempo Moderno en la conformación de lo que M. Fumaroli ha llamado el Estado Cultural.

Según la idea del filósofo francés se trata de una compartimentación de la esfera cultural que al ser gestionada únicamente desde el poder garantiza la supervivencia del Estados moderno y a través de su mecánica subsidiaria la dependencia de sus súbditos.

Podemos decir que la preocupación 'moderna' por esta razón intemporal, presumiblemente, antigua como la humanidad misma, cuando no consustancial a la propia conducta humana ${ }^{2}$, tiene su origen, como tantas otras, en ese periodo de recopilación y recomposición de los saberes que fue la Ilustración y su todavía vigente caudal de conocimientos.

\footnotetext{
${ }^{2}$ Dicho sea, esto, con la mayor de las precauciones, pero todas las teorías que preconizan que la conducta humana no es más que una especie de condensación de experiencias y sabidurías acumuladas que van muy poco a poco fijándose en los genes, que finalmente la transmiten, no están lejos de esta idea. Con seguridad, los primeros hombres ya heredaban los objetos que habían pertenecido a otros, y sin duda la sabiduría que habían generado. Y en cualquier caso, los enterramientos rituales con ajuares pertenecientes al difunto no hacen más que evidenciar esta certeza. Para la primera idea, ver, por ejemplo, Cairns, R. B.; Elder, G. H. Jr. \& Costello, E. J. (Eds.): Developmental Science. Cambridge, Cambridge University Press, 1996 O Elman, J. L.; Bates, E. A.; JOHNSON, M. H.; Karmiloff-Smith, A.; Parisi, D. \& Plunkett, K.: Rethinking Innateness. A Connectionist Perspective On Development. MIT Press, 1996.
} 
Durante la Asamblea constituyente que gobernó Francia entre 1792 y 1795, conocida como la Convención, el famoso Abate Grégoire, considerado el padre del sentido moderno del patrimonio, aseguraba que «le respect public entoure particulièrement les objets nationaux qui, n'étant à personne, sont la propriété de tous [...] Tous les monuments de sciences et d'arts sont recommandés à la surveillance de tous les bons citoyens» ${ }^{3}$.

Curiosamente, esta nueva dimensión 'pública' nacida en el seno del ideario de la Revolución entronca con algunos de los postulados más clásicos sobre el bien común y las obligaciones de la polis. Por esas mismas fechas (1796), AntoineChrysostome Quatremère de Quincy, en sus Cartas a Miranda4, instaura definitivamente lo que pronto se convertirá en un nuevo paradigma acerca de esa dimensión de lo patrimonial.

El autor, en una contundente denuncia de las prácticas napoleónicas de expolio patrimonial, desgrana las características modernas del Patrimonio en una reivindicación del derecho de los pueblos a su propiedad, lo cual, según su punto de vista, garantizará la libertad y el cosmopolitismo de "las artes y de las ciencias", del saber como testigo e instrumento de "civilización"; en definitiva 'república' que tiene como objeto, fin y referente "la Connaissance humaine". Quatremère de Quincy comprende que el expolio artístico italiano por parte de Napoleón no se limita a un deleznable hecho de guerra, ni a un escarmiento de los vencedores sobre los derrotados: es, en un sentido mucho más profundo, una violación del sentido nacional, un robo de la identidad, un ultraje de aquello que en mayor medida puede unir a un pueblo.

En este sentido, Quatremère de Quincy ya detecta cuáles son los atributos modernos de patrimonio, a la vez que abre la puerta a una gran teoría de la relación patrimonio/Estado. Y, sin embargo, evidencia una de las paradojas de la cultura moderna, pues concibe las artes y las ciencias como expresiones universales del genio humano, 'patrimonio' de todos los hombres y mujeres independientemente de dónde y en qué tiempo habiten, y el expolio, la desatención o la destrucción de éste perjudica tanto a la parte donde se encuentre como, por su sentido, a la humanidad entera.

\footnotetext{
${ }^{3}$ En una traducción muy libre vendría a significar que "el interés público privilegia particularmente los 'objetos' nacionales que sin ser de nadie son propiedad de todos [...] Al buen ciudadano se encomienda la vigilancia (la protección) de las grandes obras de las ciencias y las artes".

${ }^{4}$ En el original, Lettres à Miranda sur le déplacement des monumentos d'Art de l'Italie, publicado de forma anónima y clandestina, pues se trataba de una valiente reacción a la práctica de expoliación que el joven Napoleón estaba poniendo en práctica en Italia y en otros países en consecuencia con sus aspiraciones imperialistas, se recoge la correspondencia dirigida al prócer venezolano y héroe de la Revolución Francesa, el general Francisco de Miranda.
} 
En esta senda teórico histórica se va construyendo la moderna relación que señalo más arriba, y como derivación inmediata de ello, la aspiración de muchos pueblos que ven en el patrimonio en referente ideal para la reivindicación de su propia categoría de Nación, en los casos en que las circunstancias históricas hubieran impedido esa realidad.

Las naciones-Estado, por su parte, se afianzan en una atención a sus monumentos históricos que viene a equipararse en importancia fundacional al propio territorio patrio. Eminentes hombres de la cultura, la ciencia y la intelectualidad, ocupan altos cargos en lo que ya podemos llamar sin ambages camino hacia la constitución del Estado cultural.

Prosper Mérimée ocupó l'Inspection générale des monuments historiques francesa el largo periodo que media entre 1834 y 1870, y es nada menos que el filósofo Henri Bergson quien desarrolla la idea de 'patrimonio cultural' cuando en 1921 participaba en el nacimiento de la Commission internationale de la coopération intellectuelle, antecedente de la Unesco. No hay que esperar demasiado para que irrumpa en la escena André Malraux, quien en la delicada situación europea de entreguerras declaraba, en una nueva vuelta de tuerca, que "l'heritage ne se trasmet pas, il se conquiert"; el general De Gaulle lo nombrará responsable del primer Ministerio de Cultura en 1858, después de haberlo sido del Interior.

A partir del inicio de la década de los años 70 del pasado siglo, el tándem Patrimonio/Estado se constituye como ideario político principal, se trata de la nueva civitas, la cualidad de la ciudadanía moderna. El Estado, en adelante, desarrollando la idea ilustrada de bons citoyens, se convierte en garante de los derechos herenciales que asisten a sus miembros y lo consolidan a él. El Patrimonio es, cómo proclama aquella idea, de nadie pero de todos, y el nuevo Estado -ahora moderno y cultural más que ilustrado- lo va a llevar a una 'rentabilidad' por encima de la mera obligación de vigilancia y protección que para con él debe tener la 'buena ciudadanía': la cohesión y el desarrollo de los pueblos se mira en su patrimonio.

Velar por la integridad, defensa, distribución y restitución, la definición y activación, su tratamiento y protección va a ser una encomienda 'sagrada' del nuevo Estado, que hace suyas las premonitorias palabras de A. Malraux, que suenan a reto "la ciudadanía ha de ganarse su derecho patrimonial", pero que realmente reproducen aquellas felicitaciones efusivas del patrón al jornalero que aplaude y jalea lo bien y lo mucho que éste trabaja.

El nuevo Estado se convierte en subsidiario y administrador de los patrimonios que la ciudadanía se gana, con una rentabilidad política clarísima, pero nunca va a bajar a la arena de la mediación ni de la negociación (Padiglione 1999, p. 24); (Portelli, 2007, p. 
109) ${ }^{5}$. Es decir, según esta idea, los ciudadanos son depositarios/propietarios de su herencia cultural pero sólo en cuanto que forman parte de un Estado, que, como soberano, les otorga este derecho. Pero nunca al contrario, es la herencia cultural todas las herencias- común el cohesionante de un futuro Estado. $\mathrm{O}$ ¿es que acaso si no hay Estado no existe la herencia patrimonial?

Desde el inicio, pues, de la década arriba indicada, el sentido de patrimonio se alarga, se extiende. En adelante no se va a limitar estrictamente a elementos monumentales remarcables, arquitectónicos y/o artísticos, sino que irá paulatinamente dando acogida a otros patrimonios que van a conformar una especie de idea holística de lo patrimonial: los textos y documentos históricos y literarios, las lenguas locales, las músicas de tradición oral, las artesanías... y hasta la fauna y la flora, y el paisaje.

Francia, es sin duda el país en el que más se avanza en este nuevo sentido del patrimonio. En agosto de 1978 el ministerio de cultura crea una dirección general de patrimonio, que abre las puertas a la muy prestigiosa Misión du Patrimoine Ethnologique (1980). A la par, también es en Francia donde surgen las más ponderadas críticas a la labor del Estado cultural y a la administración del patrimonio como mecánica subsidiaria ${ }^{6}$.

De forma paralela a esta reconversión del Estado, la UNESCO aprueba, en noviembre de 1992, la Convención sobre la protección del patrimonio mundial cultural y natural, bajo declaraciones y auspicios de buena voluntad y armonía: "La declaración de un bien patrimonio de la humanidad es un privilegio y un prestigio para el país, para sus responsables políticos y culturales y para los ciudadanos más directamente relacionados con el referido Bien Patrimonio de la Humanidad. Pero al mismo tiempo es una gran responsabilidad, ya que deben mantener su conservación y protección así como profundizar en su estudio con el fin de que su Bien pueda contribuir a la educación integral de otras personas y pueblos" 7 .

\footnotetext{
5 En el mismo sentido, ver también la interesante aportación de CLEMENTE, P.: "Negociar la diversidad...", en: GARCÍA, M. y PALACIOS, J. (eds.) Patrimonio cultural. Nuevas formas de tratamiento/nuevos sentidos. Sphera Publica n ${ }^{\circ}$ especial, Murcia, Quaderna-Ucam.

${ }^{6}$ Es ingente $y$, por otro lado, bastante desconocida, o podría decirse, con más tino, que poco relacionada en contextos de interés con el asunto principal que trata este texto, la producción crítica francesa, de la que apuntaré al menos dos nombres y cuatro obras que me parecen muy centradas. De LENIAUD, Jean-Michel : L'Utopie française, essai sur le patrimoine (Mengès, Paris, 1992) y Les archipels du passé: le patrimoine et son histoire (Fayard, Paris, 2002); de POIRRIER, Philippe: L'État et la culture en France au XXe siècle (Le Livre de Poche, Paris, 2006) y como editor, Politique culturelle et patrimoines, Culture \& Musées, juin 2007 n9. El primero, quizá en la senda marcada por el indispensable El culto moderno a los monumentos, de Aloïs RIEGL (varias ediciones en español: Visor 1987, 1999; La Balsa de la Medusa 2008; IAPH 2007...).
}

7 Ver en: http://www.patrimonio-mundial.com/unesco1.htm 


\subsection{El Patrimonio desde dentro}

Es muy posible que entre las razones que justifican esta resignada $-y$ enormemente generalizada- aceptación del Patrimonio como incontestable fenómeno sociocultural y económico de nuestro tiempo se encuentre, además de las ya citadas y evidentes, la que lo asume como una compensación, una especie de neutralización que equilibra y contrarresta el evidente desamparo en que el individuo integrante de esta postmodernidad hedonista y de capitalismo salvaje se encuentra frente a los grandes monopolios políticos, informativos y económicos.

Cumpliría así una de las expectativas que más se agradecen entre las posibles que definen Patrimonio: la del reconfortamiento sentimental. Frente a la evidencia del triunfo incuestionable de las leyes de libre mercado ante las que ni siquiera el Estado parece poder garantizar la defensa del ciudadano, tanto el propio Estado como algunas importantes instituciones financieras o grandes empresas 'ofrecen' una especie de panacea que se resuelve en una metafórica propiedad de las grandes obras de la humanidad o ahora ya también de las pequeñas cosas que desde la perspectiva social propician un consuelo cultural que no va en realidad más allá de una posesión absolutamente virtual.

Sin embargo las adhesiones a las filas de la militancia patrimonial son contundentes, casi de júbilo incontenido, entusiastas. El sentido optimista del Patrimonio por el que todo el mundo -nunca mejor dicho en los casos de Patrimonio de la Humanidad- se siente feliz 'propietario' aunque sea metafórica y virtualmente de las mayores riquezas y tesoros arrastra una incontenible muchedumbre enardecida por esta nueva forma de posesión. El proselitismo se convierte, en este contexto, en la expresión más acabada de la voluntad de los pueblos, que ya no pretenden revoluciones, sino protección, salvaguarda, defensa... de sus patrimonios materiales, monumentales e intangibles.

Toda una declaración de intenciones, cuando no un manifiesto ideológico, podemos encontrar justo en el marco de estudios propuesto para la reunión científica que da cobijo a esta comunicación. En la entradilla resumen y de reclamo publicitario de un libro dedicado nada menos que a la comunicación global del patrimonio dice textualmente que se reflexiona sobre los elementos que propician la plena democratización del acceso a nuestro patrimonio cultural y se analizan, desde una perspectiva multidisciplinaria, aspectos como la interpretación, la didáctica y los medios que aseguran la plena accesibilidad emocional e intelectual al patrimonio cultural, sin menoscabar por ello la solidez científica.

Realidades como la difusión preventiva -que permite equilibrar el uso y la preservación-, la publicidad, la comunicación gráfica o las relaciones públicas -que facilitan una conexión efectiva entre los potenciales usuarios y los recursos patrimoniales activados, asegurando la maximización de los recursos humanos y 
materiales- o el papel cada vez más relevante de las tecnologías de la información y la comunicación son abordadas desde una visión comunicativa global.

La mirada teórica y analítica que a estos aspectos dirige la obra se realiza con un enfoque actualizado respecto a los desafíos que plantea el nuevo siglo, en el que los bienes culturales se han transformado en potentes activos que deberían estar al servicio del conjunto de la sociedad. En este contexto, la comunicación global se presenta como un componente fundamental que permite un conocimiento atractivo, sostenible y eficiente de nuestro patrimonio cultural.

Es decir, se hará todo lo que se pueda para que esa realidad de bondad incontenible, de desarrollo armónico del conjunto de la sociedad y de acceso plenamente democrático de sus integrantes se convierta -eso sí, bajo una difusión preventiva- en un óptimo disfrute y una conexión efectiva emocional e intelectual.

Sin embargo, no sabemos si 'la mirada analítica' aclarará de quién o de qué hay que defender al Patrimonio. Si de nosotros mismos, que a veces inexplicablemente lo agredimos sin sombra de misericordia; de los temibles envites de promotores, constructores y demás ralea, que agraden con más contundencia cuando mejor sean los sitios objeto de su codicia; o de las propias Instituciones que puede que en un momento dado no tengan claro o no oigan con la suficiente nitidez las soflamas reivindicativas que de tal o cual patrimonios hacen las gentes.

El párrafo literal reproducido más arriba nos da una de las claves más repetidas en la literatura crítica acerca de esa imposibilidad real de mirar desde afuera el Patrimonio. Como he mantenido anteriormente la enorme importancia socioeconómica del Patrimonio engulle el fenómeno en su totalidad haciendo imposible la percepción de voces críticas, o de alertas de complacencia hedonista e interesada en este universo de las herencias culturales.

Indudablemente, nuestro tiempo y nuestra sociedad se encuentran en la encrucijada del vértigo. Los cambios son enormemente profundos y se producen con una rapidez inaudita. La ya clásica idea de R. Koselleck (2003), que introduce la noción hasta entonces poco o nada atendida de 'aceleración' en el análisis del cambio social, adquiere plena relevancia en la actualidad.

En efecto, la característica esencial de los cambios en nuestra sociedad es la del vértigo acelerativo. Entre el encumbramiento del tratamiento patrimonial como fenómeno privilegiado de la modernidad tardía y su desintegración entre los referentes que 'verdaderamente interesan' entre las nuevas maneras comunicativas globales, no media ni tiempo ni espacio.

El Patrimonio hoy puede ser detectado entre las políticas cosmopolitas y transnacionales de las instituciones mundiales a la espera paciente de su consideración como bien de interés; el debate un tanto trasnochado de su poder de condensación simbólica y de referente de identidad de 'los pueblos', realmente disuelto en sus 
posibilidades reales como recurso turístico -y por lo tanto, económico-; y una helada indiferencia social en las estrategias comunicativas globales.

Estamos en la era, en una época en la que el peor destino de cualquier asunto social no lo suponen las críticas aceradas o los levantamientos sociales contrarios sino la indiferencia que por él muestren las redes sociales.

Da la impresión de que por un lado marchan las grandes políticas sobre el patrimonio y su corte de adeptos, y por otra, en un paralelismo desesperante y nunca convergente, el 'verdadero' interés social, que en casi ningún caso va más allá del interés meramente turístico.

Los asuntos relacionados con el patrimonio son la hermana pobre de la notable convulsión que los medios de comunicación experimentan en los últimos tiempos con la irrupción de las modalidades comunicativas de las nuevas tecnologías digitales. Si se utiliza, se reduce casi siempre a la denuncia de deterioro, de atentado, que parece ser lo verdaderamente noticiable, y en cuyo caso 'conviene' acto seguido desatar toda una soflama de enaltecida lucha por la supervivencia y encendida defensa de sus valores.

Si no, en el lado absolutamente contrario, se alaba, se divulgan los beneficios de su conocimiento y de su disfrute turístico, llegándose incluso, como ya hemos visto que sucede en la actualidad, a una especie de ranking en la que una zona o una ciudad sin uno o más patrimonios declarados (a ser posible con la vitola de Patrimonio de la Humanidad) difícilmente va a entrar a formar parte de las preferencias de visita turística. Aún así, en la prensa -o en otros medios modernos on-line- una de las bazas destacadas es el desvelamiento de la enjundia de patrimonios olvidados.

Definitivamente se vislumbran dos grandes corrientes en lo que al tratamiento teórico del patrimonio se refiere: una lo entronca en una serie envolvente de procedimientos sociales que solo tiene virtudes: construir el futuro desde la perspectiva del pasado, constituirse como referente de identidad de los grupos sociales, dinamizar y rentabilizar los lugares constituyéndose en la modernidad tardía y en tiempos de crisis en casi la única alternativa de desarrollo, objetivo de prestigio y eufemismo de exclusivismo. Es lo que pudiéramos llamar la versión optimista.

Frente a ésta, se sitúan las posiciones críticas que, de forma muy generalizada, pueden resumirse en tres grandes líneas: la construcción del estado cultural, el 'malestar' del patrimonio (segregación, imposición, etc.) y arbitrariedad de las políticas culturales (que lleva a una especie de espiral competitiva para alcanzar las glorias patrimoniales). 
El Patrimonio es en nuestros días inequívoca y fundamentalmente una apuesta de desarrollo, un asunto que no va más allá de lo comercial, en cuyo caso las referencias a su dimensión de confortamiento social en el sentido de su presunto poder de condensación simbólica y/o identitaria funcionan únicamente como justificación o como eximente de la arbitrariedad que es componente esencial de la consideración patrimonial institucional.

Así, el asunto convertido en una perspectiva de futuro utiliza sus pretendidos étimos herenciales, y por lo tanto como dialéctica del pasado, para forzar una impresión social reconfortante. Ese es el motivo fundamental por el que sólo se consideran patrimoniales los 'bienes', las herencias más bondadosas y 'positivas' y no las deleznables y/o negativas, aun cuando éstas son tan herencia cultural como las otras, y sin duda alguna ayudarían de igual manera a la construcción y a la preservación de la memoria colectiva. ${ }^{8}$

\subsection{La comunicación, la información cultural y el Patrimonio}

Desde que los grandes de la Escuela de Frankfurt, a partir de los años 30 del pasado siglo, iniciaran su particular visión crítica acerca de la participación de los modernos medios de comunicación masiva (por aquel momento, cine y radio fundamentalmente) en la construcción de un nuevo universo simbólico de referencia la cultura de masas-, las implicaciones teóricas entre los campos informativo y cultural han experimentado un desarrollo cualitativo trascendental. Las aportaciones, en especial en el último tercio del siglo $\mathrm{XX}$, primero de los denominados Estudios Culturales -originales de la Escuela de Birmingham- y, posteriormente, de la perspectiva de las mediaciones desde el ámbito latinoamericano, han acabado por revalorizar definitivamente el proceso de la información como un anclaje desde el que reinterpretar las relaciones sociales y culturales.

A ello hay que añadir, necesariamente, el impacto de las tesis y resultados de estudio de la Escuela de Palo Alto, en lo que a la propia naturaleza de la comunicación y sus medios respecta. Grandes teóricos han definido a la sociedad contemporánea como la sociedad de la información, aunque quizás fuera más oportuna la denominación de sociedad de las tecnologías de la información. En medio de todo ello late una preocupación también muy contemporánea pero verdaderamente muy poco extendida como es la que ha de plantear matices de distinción entre información y comunicación, si bien como digo está muy generalizada una confusión entre ambas

\footnotetext{
${ }^{8}$ En honor a la verdad, también se han activado Patrimonios en este último sentido: la Memoria del Holocausto, Hiroshima, la Ley de la Memoria Histórica, etc. Pero siguen siendo casos/elementos extremos que fundamentalmente adquieren como función resaltar los avances sociopolíticos alcanzados en la superación de etapas o de hechos, para la inmensa mayoría, nefastos. revalorizar definitivamente el proceso de la información como un anclaje desde el que reinterpretar las relaciones sociales y culturales.
} 
realidades.

La comunicación es una faceta humana que va mucho más allá de lo puramente informativo. El significado de comunicar [del latín vulgar (circa 1440) communicare < com-munere (de donde comunis) < munere (orig. moneo)] debería estar más cerca de 'compartir' -incluso como lo estuvo en la Edad Media, de comulgar-, en cualquier caso de hacer -en el sentido de elaborar o llevar a cabo- u organizar cosas en común. Aunque es posible hablar de la comunicación en los animales, pues como se sabe hay muchos de ellos capaces de transmitirse cierta información, sus estrategias son siempre instintivas y pautadas según un código de comportamiento no racional. Tal y como hoy la entendemos, la comunicación es una facultad específicamente humana, una cuestión ontológica y un hecho diferencial con el resto de criaturas. Más allá del mero intercambio o transmisión de ideas, la comunicación conforma una especie de atmósfera -en sentido metafórico- fuera de la cual es imposible la existencia humana.

Es decir, puede asumirse que según la teoría de los filósofos del lenguaje (fundamentalmente Wittgenstein y Heidegger), lo lingüístico es el universo humano, y en ese mismo sentido lo comunicacional es exactamente ese mismo universo, esa especial atmósfera, como acabo de llamarle. Como en su día expresara el dictum d Palo Alto "es imposible no comunicar".

El concepto de cultura es igualmente inabordable por lo complejo, ambiguo y extenso de sus acepciones y significados. Desde el punto de vista antropológico, cultura puede ser considerado en idéntico significado que comunicación. Así, añadiendo más matices a la dimensión específicamente humana de comunicar, J. Martín-Barbero (1990) precisa que se trata de "hacer posible que unos hombres reconozcan a otros, y ello en doble sentido: les reconozcan el derecho a vivir y pensar diferentemente, y se reconozcan como hombres en esa diferencia. Eso es lo que significa y lo que implica pensar la comunicación desde la cultura".

Dejando en su lugar de premisa del pensamiento estas cuestiones ontológicas del lenguaje, la comunicación y la cultura, hay que abordar las funciones y las dimensiones que estos asuntos han adquirido en nuestra sociedad y cómo cada una de ellas ha devenido hacia una especial acepción y un tratamiento sui generis. Así, cultura se circunscribe casi a una especificidad, una esfera de la vida social muy ligada a las industrias culturales o en cualquier caso convenientemente diferenciada de otros aspectos de la vida como la economía, la política, etc.

La cultura pasa a constituirse en nuestro tiempo como un determinado comportamiento en cierta forma paralelo a las 'grandes cuestiones' pero relegada como a un segundo término, más elitista y sobre todo menos trascendental de nuestra sociedad. En este contexto se ubica el Patrimonio, si bien su dimensión rentabilista lo sitúa en un intersticio extraño y especial, pues por una parte se asume como cultura y por otra como ya se he visto cumple una serie de importantes 
estrategias politicoeconómicas.

Por su parte, la comunicación se ha ido identificando con la labor que desarrollan los medios -por ello precisamente llamados de comunicación-. La cuestión crucial de este asunto es la imposibilidad de distinguir adecuadamente información y comunicación.

Contra la idea macluhaniana de la comunicación como un utensilio, como un mero medio, en las nuevas teorías su naturaleza se versiona como encrucijada que construye, que hace realidad, que se torna imprescindible e intrínsecamente necesaria para ese gran objetivo de la convivencia y connivencia de la-s cultura-s (en esto radica su grandeza, su enorme poder y todos los 'peligros' que lógicamente de todo ello se desprende).

Asumiendo que la de los medios es una labor excelsa, pro social, puede decirse que "probablemente no nos dirán qué debemos sentir o pensar de nuestro patrimonio, pero desde luego nos van a delimitar sobre qué patrimonio debemos sentir o pensar" (Sanjuán Ballano, 2007. p. 38).

Sin embargo, desde el punto de vista de que el sentido moderno de Patrimonio poco tiene que ver con la percepción o con la interiorización que personal e individualmente podemos formarnos de él, sino que por el contrario el Patrimonio se 'decide' o en el mejor de los casos se propone y se consensúa -casi siempre- desde arriba, la aseveración anterior queda en entre dicho, pues precisamente aparte de la mera labor informativa que los medios cumplirán dando cuenta de qué patrimonio se considera como tal y qué patrimonio no cuenta con la bendición institucional, sí que influirán determinantemente en "qué debemos sentir y pensar" del patrimonio. Esa es la cuestión crucial en la relación perceptiva y después dialógica que debe entablarse entre los individuos y 'su' patrimonio.

Resumiendo, un individuo poco tiene que decir acerca de lo que es o no patrimonio; en cualquier caso, y en el mejor de ellos, podrá participar de una manera más o menos metafórica en una especie de 'negociación' sobre el asunto; luego, lo que, digamos, es modulable en la opinión pública es precisamente la cualidad del Patrimonio sobre cuya catalogación como tal no hemos podido decidir: lo que está en disposición de hacer la proyección que los medios hagan de un patrimonio ya constituido institucionalmente como tal es la de ratificar una idea social sobre él.

Abordemos finalmente el fondo de lo que estas páginas proponen, esto es, cómo proyectan y divulgan los medios las cuestiones relacionadas con el Patrimonio, y por tanto, entonces, cómo influyen o cómo modelan la percepción que las personas elaboran o interiorizan de su propia herencia cultural e histórica. En otros términos quizá más genéricos: ¿cómo se ocupan los medios de la información cultural? 
García Canclini (2007), contesta que

una de las novedades que presentan los diarios en la última década es que se ha expandido el campo cultural [...] reformulación del concepto de cultura, asociada a su nuevo papel en procesos socioeconómicos y políticos donde no estábamos habituados a encontrarla [...] las diferencias derivan de los modos de organizar lo local, lo nacional y lo global en diversos campos culturales (...).

Las estrategias manejadas en las distintas secciones parecen colocar a los diarios en una disyuntiva: en la medida en que se expanden hacia lo global considerándolo como espectáculo y como lugar de negocios espectaculares, debilitan su razonamiento sobre lo público, o lo limitan a la cultura de élite y a su repercusión doméstica.

No es un problema exclusivo de los periódicos, pero tal vez sea el campo comunicacional en que se percibe con más fuerza la tensión entre la cultura como elaboración crítica y como entretenimiento".

Lo cierto es que la proyección/divulgación que de estas cuestiones hacen los medios, la mayoría de las ocasiones, se debate entre la información institucional y las diversas lecturas que las instituciones hacen de la mecánica patrimonial. Es evidente que todo lo relacionado con el 'descubrimiento' de los patrimonios más inauditos cuenta con una incondicional, cuando no entusiasta, acogida por parte de los receptores, y es evidente lo atractivo que resulta su abordaje por parte de los medios.

Digamos que, convertidas en una moda, las sugerencias turístico gastronómicas y de visita turístico patrimonial, disfrutan hoy de un específico y muy generalizado tratamiento. De otro lado las agresiones a los bienes patrimoniales -vengan de donde vengan-, los olvidos, la despreocupación, etc., se convierten fácilmente en un recurso mediático óptimo, en una noticia con 'gancho'. Todo ello viene a confirmar lo ya dicho: la relevancia del lado de viabilidad rentable o el de 'malestar' social como casi únicas estrategias de acción -ya de tratamiento como de divulgación-.

No es en absoluto tarea sencilla reflexionar sobre cómo debe de ser, entonces, el tratamiento de la cultura por parte de los medios de comunicación ni de cómo puede realmente versionarse o llevarse a cabo la idea de García Canclini sobre un razonamiento de lo público, y mucho menos sobre la elaboración crítica de la cultura. Da la sensación de tratarse de un sofisma teórico irresoluble.

Pero si de verdad pensamos en los medios como potencial de negociación social -en 
el sentido que antes he dado al término-, o progresamos en su percepción como una parte, indispensable pero parte al fin y al cabo, del universo comunicacional humano, y somos así conscientes de su enorme poder como estrategia de modelación de las mentalidades, hemos de convenir en que, de la forma que fuese, los medios tendrían que trascender lo meramente noticiable para acercarse a lo "profundamente humano" (Sanjuán Ballano, 2007, p. 38), o en cualquier caso ir más allá del sencillo mecanismo de integrar lo simple en lo complejo, y buscar un compromiso en el que lo profundamente humano tenga una armonización crítica con lo 'perfectamente' social.

En principio, por ejemplo, provocando la ruptura de una de las cadencias más significativas de la Modernidad -y, en realidad, de todos los tiempos- en el campo de la comunicación, que como hemos visto es el único y específicamente humano, cual es el del dominio absoluto o de la radical preponderancia de los emisores -las grandes conformaciones históricas de sentido social: la política, el arte y la literatura, el derecho y la justicia, la ciencia...- frente a los históricamente resignados receptores.

\section{REFERENCIAS}

Clemente, P. (2010). Negociar la diversidad. En Patrimonio cultural. Nuevas formas de tratamiento/nuevos sentidos. Murcia: Quaderna-Ucam. Murcia.

Clifford, J. (2003). Tradicional Futures. En Questions of Tradition. Toronto: Univ. of Toronto Press.

Clifford, J. (2004). Looking several ways. Anthropology and Native Heritage in Alaska. Current Anthropology, 45(1).

Cobo De Guzmán, F. (2010). La economía política de la patrimonialización y los grupos subalternos. Crítica cultural desde una etnografía plurilocal. En Patrimonio cultural. Nuevas formas de tratamiento/nuevos sentidos. Murcia: Quaderna-Ucam. Murcia.

Fumaroli, M. (1992). L'Etat culturel, essai sur une religion moderne. Paris: Editions de Fallois.

García Canclini, N. (2007). ¿Cómo se ocupan los medios de la información cultural?. Etcétera. Recuperado el 22 de marzo de 2011, de www.etcetera.com.mx/cancli.asp

Højrup, T. (2010). Del Recuerdo a la Experiencia. Herencia cultural y ambiente cultural en Dinamarca. En Patrimonio cultural. Nuevas formas de tratamiento/nuevos sentidos. Murcia: Quaderna- Ucam.

Koselleck, R. (2003). Aceleración, prognosis y secularización. Valencia: Pre-textos.

Martín-Barbero, J. (1987). De los medios a las mediaciones. Comunicación, cultura y 
hegemonía. Barcelona: Gustavo Pili.

Martín-Barbero, J. (1990). Comunicación, campo cultural y proyecto mediador. Diálogos de la comunicación, 26: 7-15. Lima: FLFC.

Mateos Rusillo, S. (2008). La comunicación global del patrimonio cultural: del marco teórico al estudio de casos. Gijón: Trea.

Neyret, R. (2004). Du monument isolé au 'tout patrimoine. Géocarrefour, 79(3). Recuperado el 23 de marzo de 2008, de http://geocarrefour.revues.org/index746.html

Padiglione, V. (1999). El efecto marco: las mediaciones del Patrimonio y la competencia antropológica. En Patrimonio Etnológico. Nuevas perspectivas de estudio. Sevilla: Junta de Andalucía.

Portelli, A. (2007). Dalla parte dei protagonista. Antropologia Museale, 17. Quatremère De Quincy, A-Ch. (2007). Cartas a Miranda. Murcia: Nausicaä.

Rodríguez Temiño, I. (1998). La tutela del Patrimonio artístico de la modernidad a la posmodernidad. Boletín del Instituto Andaluz del Patrimonio Histórico.

Sanjuán Ballano, B. (2007). Patrimonio cultural y medios de comunicación. Cuadernos PH.

\section{Modesto García-Jiménez}

Licenciado en Filología Hispánica y Doctor en Antropología Social y Cultural. Director del Área y de la Titulación de Antropología Social y Cultural de la Universidad Católica de Murcia (UCAM). Ha ejercido como documentalista del CTI (Universidad de Málaga) y la Consejería de Educación de la Junta de Andalucía. Ha realizado estancias de intercambio de profesores investigadores en las universidades Marc Bloch de Strasbourg (Francia), Basel Miembro numerario (Suiza) y Roma I “La Sapienza”. de la AEGPC, de Fundación UNESCO-Patrimonio Universidad y del CEIPatri. Ha participado en numerosas reuniones científicas y congresos internacionales y es autor de un buen número de artículos y libros. 